\title{
ON THE STABLE PROPERTY OF PROJECTIVE DIMENSION
}

\author{
SOMAYEH BANDARI AND RAHELEH JAFARI
}

\begin{abstract}
We introduce the concept of monomial ideals with stable projective dimension, as a generalization of the Cohen-Macaulay property. Indeed, we study the class of monomial ideals $I$, whose projective dimension is stable under monomial localizations at monomial prime ideals $\mathfrak{p}$, with height $\mathfrak{p} \geq \operatorname{pd} S / I$. We study the relations between this property and other sorts of Cohen-Macaulayness. Finally, we characterize some classes of polymatroidal ideals with stable projective dimension.
\end{abstract}

\section{INTRODUCTION}

Throughout the paper, $S=K\left[x_{1}, \ldots, x_{n}\right]$ denotes the polynomial ring in $n$ indeterminates over an arbitrary field $K$. Let $M$ be a finitely generated graded $S$-module. Then

$$
\left\{\mathfrak{p} \in \operatorname{Supp}(M): \operatorname{pd} M=\operatorname{pd} M_{\mathfrak{p}}\right\} \subseteq\{\mathfrak{p} \in \operatorname{Supp}(M): \text { height } \mathfrak{p} \geq \operatorname{pd} M\},
$$

where $\operatorname{pd}(-)$ denotes the projective dimension (cf. Lemma 1.1). If $M$ has positive depth, then the equality holds in (1), precisely when $M$ is Cohen-Macaulay (cf. Proposition 1.2). If we replace $M$ with $S / I$, for a monomial ideal $I$ in $S$, it is natural to consider only monomial prime ideals in $\operatorname{Supp}(S / I)=V(I)$. The monomial localization of a monomial ideal $I \subset$ $S$ at a monomial prime ideal $\mathfrak{p}$, is the monomial ideal $I(\mathfrak{p}) \subseteq S(\mathfrak{p})=K\left[x_{i} \quad: \quad x_{i} \in \mathfrak{p}\right]$ obtained from $I$ by setting $x_{i}=1$, for all variables $x_{i} \notin \mathfrak{p}$. Note that $\operatorname{pd}(S(\mathfrak{p}) / I(\mathfrak{p}))=$ $\operatorname{pd}(S / I)_{\mathfrak{p}}$ (cf. Remark 1.4). Let $V^{*}(I)$ denote the set of monomial prime ideals $\mathfrak{p}$ in $V(I)$. Example 1.6, provides a monomial ideal $I$, with $\operatorname{depth}(S / I)>0$, that satisfies

$$
\left\{\mathfrak{p} \in V^{*}(I): \operatorname{pd} S / I=\operatorname{pd}(S(\mathfrak{p}) / I(\mathfrak{p}))\right\}=\left\{\mathfrak{p} \in V^{*}(I): \text { height } \mathfrak{p} \geq \operatorname{pd} S / I\right\}
$$

but $S / I$ is not Cohen-Macaulay. The main interest in this paper, is to study the class of monomial ideals $I$, with property (2). In other words, we study the class of monomial ideals $I$ whose projective dimension is stable under monomial localizations at monomial prime ideals $\mathfrak{p}$, with height $\mathfrak{p} \geq \operatorname{pd} S / I$. In this case, $I$ is said to have stable projective dimension (cf. Definition 1.7). This class contains all monomial ideals $I$, where $S / I$ is a Cohen-Macaulay ring (cf. Proposition 1.8). Indeed the set of monomial ideals with CohenMacaulay quotient rings, is precisely the set of monomial ideals $I$ with stable projective dimension such that $\operatorname{Ass}(S / I)=\operatorname{Min}(I)$ (cf. Proposition 1.17). Taking into account that the monomial localization is a simple operation which turns out a monomial ideal in a smaller polynomial ring, the class of ideals with some stable algebraic properties under monomial localizations, have significant role in monomial algebra.

Beginning in Section 1, we study $\operatorname{Ext}^{n-t}(M, S)$, where $M$ is a finitely generated graded $S$-module with $\operatorname{depth}(M)=t$. Expressing $\operatorname{Supp}\left(\operatorname{Ext}^{n-t}(M, S)\right)$, as the set of prime ideals

2010 Mathematics Subject Classification. 13F20, 05E40.

Key words and phrases. stable projective dimension, polymatroidal ideals, monomial ideals, CohenMacaulay.

The first author was in part supported by a grant from IPM (No. 95130020). 
$\mathfrak{p} \in \operatorname{Supp}(M)$ with $\operatorname{pd} M_{\mathfrak{p}}=\operatorname{pd} M$, we specify when the equality holds in (1). Then we discuss the relations between the property of having stable projective dimension, and some weaker sorts of Cohen-Macaulayness. Several examples are included to indicate that the set of monomial ideals with stable projective dimension, is disjoint from the set of monomial ideals $I$, where $S / I$ is generalized Cohen-Macaulay or sequentially Cohen-Macaulay, and non of them contains the other. More over, it is shown that a monomial ideal $I$, where $S / I$ is a generalized Cohen-Macaulay ring, has stable projective dimension if and only if, either $S / I$ is Cohen-Macaulay or depth $S / I=0$ (cf. Proposition 1.12).

Section 2, is devoted to characterizing some classes of polymatroidal ideals who have stable projective dimension. We show that polymatroidal ideals generated in degree 2 with at least one pure power and Veronese type ideals have stability property of projective dimension. We also characterize transversal polymatroidal ideals with this property.

Several explicit examples are provided along the paper to illustrate the property of having stable projective dimension. Many of them have been computed by using CoCoA [1].

\section{Stability of PRojeCtive Dimension Under LOCALizATions}

In this paper, we consider the natural grading on the polynomial ring $S=k\left[x_{1}, \ldots, x_{n}\right]$ and all $S$-modules are graded. We use several times the Auslander-Buchsbaum formula [6, A.4.3], saying that

$$
\operatorname{pd}(M)+\operatorname{depth}(M)=n,
$$

for all finitely generated graded $S$-modules $M$. We start with the following easy lemma, that is a crucial point in our approach.

Lemma 1.1. Let $M$ be a finitely generated graded $S$-module with $\operatorname{depth} M=t$. Then

$$
\begin{aligned}
\operatorname{Supp}\left(\operatorname{Ext}^{n-t}(M, S)\right) & =\left\{\mathfrak{p} \in \operatorname{Supp}(M): \operatorname{pd} M=\operatorname{pd} M_{\mathfrak{p}}\right\} \\
& \subseteq\{\mathfrak{p} \in \operatorname{Supp}(M): \text { height } \geq \operatorname{pd} M\} .
\end{aligned}
$$

Proof. By the Auslander-Buchsbaum formula, $\mathrm{pd} M=n-t$. Since, $\mathrm{pd} M_{\mathfrak{p}} \leq \operatorname{pd} M$ for any prime ideal $\mathfrak{p}$ of $S, \operatorname{Ext}^{n-t}\left(M_{\mathfrak{p}}, S_{\mathfrak{p}}\right) \neq 0$ precisely when $\operatorname{pd} M_{\mathfrak{p}}=\operatorname{pd} M$. This provides the first equality. The inequality, follows from the fact that $\operatorname{pd} M_{\mathfrak{p}}=$ height $\mathfrak{p}-\operatorname{depth} M_{\mathfrak{p}}$.

The following result, specifies when the equality holds in (4).

Proposition 1.2. Let $M$ be a nonzero finitely generated graded $S$-module. Then the following statements are equivalent.

(a) $\operatorname{pd} M=\operatorname{pd} M_{\mathfrak{p}}$, for all $\mathfrak{p} \in \operatorname{Supp}(M)$ with height $\mathfrak{p} \geq \operatorname{pd} M$.

(b) Either $M$ is Cohen-Macaulay or depth $M=0$.

If the above conditions hold, then $\operatorname{dim} \operatorname{Ext}^{n-t}(M, S)=t$.

Proof. (a) $\Rightarrow$ (b). By Lemma 1.1, $\{\mathfrak{p} \in \operatorname{Supp}(M) ; \operatorname{height} \mathfrak{p}=\operatorname{pd} M\}=\operatorname{Min}\left(\operatorname{Ext}^{n-t}(M, S)\right)$, is a finite set. Hence either $\operatorname{pd}(M)=$ height( $(\mathfrak{p})$ for all minimal primes $\mathfrak{p}$ of $M$, or $\operatorname{pd}(M)=n$, from Ratliff's weak existence theorem [11, Theorem 31.2]. Consequently $\operatorname{depth} M=\operatorname{dim} M$ or $\operatorname{depth}(M)=0$.

(b) $\Rightarrow$ (a). If depth $M=0$, then pd $M=n$ and so the graded maximal ideal of $S$ is the only prime ideal of height greater than or equal to $\operatorname{pd} M$. Assume that $M$ is Cohen-Macaulay. Let $I=\operatorname{Ann} M$. Then $M_{\mathfrak{p}}$ is Cohen-Macaulay, for all $\mathfrak{p} \in \operatorname{Supp} M$ and

$$
\operatorname{pd} M_{\mathfrak{p}}=\text { height } \mathfrak{p}-\operatorname{dim} M_{\mathfrak{p}}=\operatorname{height} I_{\mathfrak{p}}=\operatorname{height} I=\operatorname{pd} M,
$$


which implies (a). For the last statement, let $J=\operatorname{Ann}\left(\operatorname{Ext}^{n-t}(M, S)\right)$ and $I=\operatorname{Ann} M$. Then

$$
I \subseteq J \text { and height } I \leq \operatorname{pd} M \text {. }
$$

If $\operatorname{pd} M<$ height $J$, then there exists $\mathfrak{p} \in \operatorname{Supp}(M)$ such that

$$
\operatorname{pd} M \leq \text { height } \mathfrak{p}<\text { height } J .
$$

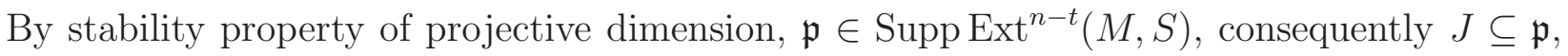
a contradiction. Hence pd $M \geq$ height $J$ and so

$$
\operatorname{pd} M=n-\operatorname{depth} M \leq n-\operatorname{dim} \operatorname{Ext}^{n-t}(M, S)=\text { height } J \leq \operatorname{pd} M,
$$

which completes the proof.

Corollary 1.3. Let $M$ be a nonzero finitely generated graded $S$-module. If $\mathrm{pd} M=\operatorname{pd} M_{\mathfrak{p}}$ for all $\mathfrak{p} \in \operatorname{Supp}(M)$ with height $\mathfrak{p} \geq \operatorname{pd} M$, then $\operatorname{Ext}^{n-t}(M, S)$ is a Cohen-Macaulay module of dimension $t=\operatorname{depth}(M)$.

Proof. We have that $M$ is Cohen-Macaulay or $\operatorname{depth}(M)=0$, by Proposition 1.2. If $M$ is Cohen-Macaulay of $\operatorname{dim}(M)=t$, then $\operatorname{Ext}^{n-t}(M, S)$ is a Cohen-Macaulay module of dimension $t$, [9, Theorem 1.4]. Now, if $\operatorname{depth}(M)=0$, then $\operatorname{pd} M=n$. Hence by Lemma 1.1, $\operatorname{Supp}\left(\operatorname{Ext}^{n-t}(M, S)\right)=\{\mathfrak{m}\}$, and $\operatorname{dim}\left(\operatorname{Ext}^{n-t}(M, S)\right)=0$.

The monomial localization of a monomial ideal $I \subset S$ with respect to a monomial prime ideal $\mathfrak{p}$ is the monomial ideal $I(\mathfrak{p}) \subset K\left[x_{i}: x_{i} \in \mathfrak{p}\right]$ which is obtained from $I$ by substituting the variables $x_{i} \notin \mathfrak{p}$ by 1 .

Remark 1.4. Let $\mathfrak{p}$ be a monomial prime ideal containing the monomial ideal $I$. Then $I(\mathfrak{p})$ is the unique monomial ideal with the property that $I(\mathfrak{p}) S_{\mathfrak{p}}=I S_{\mathfrak{p}}$. For a polynomial $f \in S$, let $f(\mathfrak{p})$ denote the polynomial in $S(\mathfrak{p})$, obtained from $f$ by substituting the variables $x_{i} \notin \mathfrak{p}$ by 1 . If $I$ is an ideal in $S$ generated by monomials $u_{1}, \ldots, u_{r}$, then $I(\mathfrak{p})$ is the monomial ideal generated by $u_{1}(\mathfrak{p}), \ldots, u_{r}(\mathfrak{p})$ in $S(\mathfrak{p})$. Note that $u_{i}=u_{i}(\mathfrak{p}) v_{i}$, for some $v_{i} \notin \mathfrak{p}$. As $v_{i}$ is an invertible element in $S_{\mathfrak{p}}$, it is not difficult to see that $\operatorname{depth}(S / I)_{\mathfrak{p}}=\operatorname{depth} S(\mathfrak{p}) / I(\mathfrak{p})$ and $\operatorname{dim}(S / I)_{\mathfrak{p}}=\operatorname{dim} S(\mathfrak{p}) / I(\mathfrak{p})$. In particular $\operatorname{pd}(S / I)_{\mathfrak{p}}=\operatorname{pd} S(\mathfrak{p}) / I(\mathfrak{p})$.

The following example, shows that the converse of Corollary 1.3 is not true.

Example 1.5. Let $S=K[x, y, z, u], I=(x, y) \cap(y, z, u)$ and $M=S / I$. Then $\operatorname{depth}(M)=1$ and $\operatorname{pd} M=3$. Note that $\operatorname{Supp}\left(\operatorname{Ext}^{3}(M, S)\right) \subseteq \operatorname{Supp}(M)=V(I)$ and $(x, y)$ is the only prime ideal of height 2 , in $V(I)$. Since $\operatorname{pd}\left(M_{(x, y)}\right)=2$ and $\operatorname{pd}\left(M_{(y, z, u)}\right)=3$, we have $(x, y) \notin \operatorname{Supp}\left(\operatorname{Ext}^{3}(M, S)\right)$ and $(y, z, u) \in \operatorname{Supp}\left(\operatorname{Ext}^{3}(M, S)\right)$. In particular dim $\operatorname{Ext}^{3}(M, S)=$ $\operatorname{dim} S /(y, z, u)=1=\operatorname{depth} M$. We may also see that $\operatorname{depth} \operatorname{Ext}^{3}(M, S)=1$, using computations by CoCoA [1].

If $\operatorname{pd} M=\operatorname{pd} M_{\mathfrak{p}}$ only for monomial prime ideals $\mathfrak{p}$, with height $(\mathfrak{p}) \geq \operatorname{pd}(M)$, that is a finite set, then we don't necessarily get the statement (b) in Proposition 1.2. Here is an example:

Example 1.6. Let $S=K[x, y, z]$ and $M=S / I$, where $I$ is the monomial ideal $I=$ $x(x y, x z, y z)=(x) \cap\left(x^{2}, y\right) \cap\left(x^{2}, z\right) \cap(y, z)$. Then $\operatorname{dim} M=2, \operatorname{depth} M=1$ and $\operatorname{pd}(M)=2$. Let $\mathfrak{p} \neq \mathfrak{m}$ be a monomial prime ideal in $\operatorname{Supp}(M)$, with height $(\mathfrak{p}) \geq 2$. Then $\mathfrak{p} \in \operatorname{Ass}(M)$ and so $\operatorname{pd}\left(M_{\mathfrak{p}}\right)=2-\operatorname{depth}\left(M_{\mathfrak{p}}\right)=2$. But it does not hold for the non-monomial prime $\mathfrak{q}=(x, y+z)$. Since $M_{\mathfrak{q}}=S_{\mathfrak{q}} /\left(x^{2}\right) S_{\mathfrak{q}}=\left(S /\left(x^{2}\right)\right)_{\mathfrak{q}}$ is Cohen-Macaulay of dimension one, we have $\operatorname{pd}\left(M_{\mathfrak{q}}\right)=\operatorname{height}(\mathfrak{q})-\operatorname{depth} M_{\mathfrak{q}}=2-1=1$. 
In the sequel, we consider stability property for monomial ideals with localization in monomial prime ideals. In other words, we are interested to study the class of $S$-modules, which intersecting the sets in (4) with the set of monomial ideals, the equality holds.

Definition 1.7. A monomial ideal $I \subset S$ has stable projective dimension if $\mathrm{pd} S / I=$ $\operatorname{pd} S(\mathfrak{p}) / I(\mathfrak{p})$ for all monomial prime ideals $\mathfrak{p} \in V^{*}(I)$ with height $\mathfrak{p} \geq \operatorname{pd} S / I$.

Note that a monomial ideal $I$ has stable projective dimension if and only if

$$
V^{*}(I) \cap \operatorname{Supp}\left(\operatorname{Ext}^{n-t}(S / I, S)\right)=\left\{\mathfrak{p} \in V^{*}(I): \operatorname{height}(\mathfrak{p}) \geq \operatorname{pd} S / I\right\},
$$

where $t=\operatorname{depth} S / I$.

Proposition 1.8. Let $I \subset S$ be a monomial ideal. Then the following conditions are equivalent:

(a) $S / I$ is Cohen-Macaulay.

(b) $\operatorname{pd} S / I=\operatorname{pd} S(P) / I(P)$ for all $P \in V^{*}(I)$.

In particular, if $S / I$ is Cohen-Macaulay, then I has stable projective dimension.

Proof. (a) $\Rightarrow$ (b): Since $S / I$ is Cohen-Macaulay, then $S(P) / I(P)$ is Cohen-Macaulay for all $P \in V^{*}(I)$. Therefore

$$
\operatorname{pd} S(P) / I(P)=\text { height } I(P)=\text { height } I=\operatorname{pd} S / I
$$

for $P \in V^{*}(I)$, as desired.

(b) $\Rightarrow$ (a): We choose $P \in V^{*}(I)$ such that height $P=n-\operatorname{dim} S / I$. Then we get

$$
n-\operatorname{depth} S / I=\operatorname{pd} S / I=\operatorname{pd} S(P) / I(P) \leq \operatorname{height} P=n-\operatorname{dim} S / I .
$$

It follows that $\operatorname{depth} S / I=\operatorname{dim} S / I$. In other words, $S / I$ is Cohen-Macaulay.

Corollary 1.9. All monomial ideals in $S$ of height $n-1$, have stable projective dimension.

Proof. Let $I \subset S$ be a monomial ideal of height $n-1$. So either depth $S / I=0$ or depth $S / I \geq$ $1=\operatorname{dim} S / I$. Hence either $\operatorname{depth} S / I=0$ or $S / I$ is Cohen-Macaulay. So by proposition 1.2, $I$ has stable projective dimension.

For a monomial ideal $I$, the ring $S / I$ is called generalized Cohen-Macaulay, if $(S / I)_{\mathfrak{p} / I}$ is Cohen-Macaulay for all prime ideals $\mathfrak{p} \in V(I) \backslash\{\mathfrak{m}\}$, and $I$ is equidimensional, i.e. $\operatorname{dim}(S / \mathfrak{p})=\operatorname{dim}(S / I)$ for all $\mathfrak{p} \in \operatorname{Min} I$. Let $I$ be a monomial ideal of $S$, then the ring $S / I$ is generalized Cohen-Macaulay precisely when $I$ is equidimensional and the $\operatorname{ring} S(\mathfrak{p}) / I(\mathfrak{p})$ is Cohen-Macaulay for all monomial prime ideals $\mathfrak{p} \in V^{*}(I)$ with $\mathfrak{p} \neq \mathfrak{m}$ (cf. [3, Lemma 4.1]).

Example 1.10. Let $I$ be the ideal in Example 1.6. Then $I$ has stable projective dimension, but $S / I$ is not generalized Cohen-Macaulay, because $I$ is not equidimensional.

Example 1.11. Let $I=\left(x_{1}, x_{2}\right) \cap\left(x_{3}, x_{4}\right)$ be an ideal in $S=K\left[x_{1}, \ldots, x_{4}\right]$. Then $\mathrm{pd} S / I=3$, while $\operatorname{pd} S(\mathfrak{p}) / I(\mathfrak{p})=\operatorname{pd}\left(K\left[x_{1}, x_{2}, x_{3}\right] /\left(x_{1}, x_{2}\right)\right)=2$, for $\mathfrak{p}=\left(x_{1}, x_{2}, x_{3}\right)$. Therefore, $I$ does not have stable projective dimension. Since, any monomial prime ideal $\mathfrak{p} \in V^{*}(I) \backslash\{\mathfrak{m}\}$ contains only one of $\left(x_{1}, x_{2}\right)$ or $\left(x_{3}, x_{4}\right), S(\mathfrak{p}) / I(\mathfrak{p})$ is Cohen-Macaulay. In particular, $S / I$ is generalized Cohen-Macaulay. 
The above two examples, show that the class of monomial ideals with stable projective dimension, and the class of monomial ideals $I$ such that $S / I$ is a generalized Cohen-Macaulay ring, are disjoint. The following result, specifies the intersection of these classes. Recall that for a finitely generated $S$-module $M, \operatorname{Assh}(M)=\{\mathfrak{p} \in \operatorname{Ass}(M): \operatorname{dim} S / \mathfrak{p}=\operatorname{dim} M\}$.

Proposition 1.12. Let $I \subset S=K\left[x_{1}, \ldots, x_{n}\right]$ be a generalized Cohen-Macaulay. Then the following conditions are equivalent:

(a) I has stable projective dimension.

(b) Either $S / I$ is Cohen-Macaulay or depth $S / I=0$.

Proof. (a) $\Rightarrow(\mathrm{b})$ : Let $I$ have stable projective dimension with depth $S / I \neq 0$ which is not Cohen-Macaulay. Since $I$ is not Cohen-Macaulay, it follows that $\operatorname{pd} S / I>$ height $I$. Let $\mathfrak{p}^{\prime} \in \operatorname{Assh} S / I$ and $\mathfrak{p}=\mathfrak{p}^{\prime}+\mathfrak{q} \in V^{*}(I)$ be a monomial prime ideal with height $\mathfrak{p}=\operatorname{pd} S / I$. Since height $\mathfrak{p}=\operatorname{pd} S / I=n-\operatorname{depth} S / I<n$, it follows that $\mathfrak{p} \neq \mathfrak{m}$. Hence since $I$ is generalized Cohen-Macaulay, we have that $S(\mathfrak{p}) / I(\mathfrak{p})$ is Cohen-Macaulay. Therefore

$$
\operatorname{pd} S(\mathfrak{p}) / I(\mathfrak{p})=\text { height } I(\mathfrak{p}) \leq \text { height } I=\text { height } \mathfrak{p}^{\prime}<\text { height } \mathfrak{p}=\operatorname{pd} S / I \text {. }
$$

So $I$ does not have stable projective dimension.

(b) $\Rightarrow$ (a) is obvious by Proposition 1.2.

Remark 1.13. For a monomial ideal $I$ with height $(I)=n-1$, the $\operatorname{ring} S / I$ is a one dimensional generalized Cohen-Macaulay ring. Proposition 1.12 interprets the argument of the proof of Corollary 1.9, in a more general content.

For a finitely generated $S$-module $M$, of dimension $d$, let $M_{i}$ denote the largest submodule of $M$ such that $\operatorname{dim} M_{i} \leq i$, for $0 \leq i<d$. Note that $M_{i-1} \subseteq M_{i}$. The increasing filtration $\left\{M_{i}\right\}_{i=0}^{d}$, is called the dimension filtration. Note that Ass $M_{i} / M_{i-1}=\{\mathfrak{p} \in \operatorname{Ass} M$ : $\operatorname{dim} S / \mathfrak{p}=i\}$ (cf. [12, Corollary 2.3]). Therefore, $M_{i} / M_{i-1}$ is either zero or an $i$-dimensional module. $M$ is called sequentially Cohen-Macaulay, if $M_{i} / M_{i-1}$ is a Cohen-Macaulay $S$-module for $i=1, \ldots, d$.

Remark 1.14. Let $M$ be a finitely generated $S$-module of dimension $d$. If $\operatorname{Ass}(M)=$ $\operatorname{Assh}(M)$, then $M_{i}=0$, for $i=0, \ldots, d-1$ and $M_{d}=M$. In particular, $M$ is CohenMacaulay if and only if $M$ is sequentially Cohen-Macaulay and $\operatorname{Ass}(M)=\operatorname{Assh}(M)$.

Example 1.15. Let $S=K\left[x_{1}, \ldots, x_{4}\right]$ and $I=\left(x_{1} x_{2}, x_{2} x_{3}, x_{3} x_{4}, x_{1} x_{4}, x_{1} x_{3}\right)=\left(x_{1}, x_{3}\right) \cap$ $\left(x_{1}, x_{2}, x_{4}\right) \cap\left(x_{2}, x_{3}, x_{4}\right)$. Since $I$ is the edge ideal of a chordal graph, $S / I$ is sequentially Cohen-Macaulay, by [4, Theorem 3.2]. $R / I$ is a two dimensional ring, which is not CohenMacaulay and does not have the maximal ideal in its associated prime ideals. Therefore, $\operatorname{depth} S / I=1$ and $p d S / I=3$. Let $\mathfrak{p}=\left(x_{1}, x_{2}, x_{3}\right)$. Then $S(\mathfrak{p}) / I(\mathfrak{p})=K\left[x_{1}, x_{2}, x_{3}\right] /\left(x_{1}, x_{3}\right)$ is of projective dimension 2. Hence $I$ does not have stable projective dimension.

By definition, all monomial ideals $I$ with $\operatorname{depth}(S / I)=0$, have stable projective dimensions. But $S / I$ is not necessarily sequentially Cohen-Macaulay. Here is an example in the class of monomial ideals, with positive depth.

Example 1.16. Let $S=K\left[x_{1}, \ldots, x_{4}\right]$ and $I=\left(x_{1}, x_{4}\right) \cap\left(x_{2}, x_{3}\right) \cap\left(x_{1}, x_{2}, x_{3}\right)^{2} \cap\left(x_{1}, x_{2}, x_{4}\right)^{2} \cap$ $\left(x_{1}, x_{3}, x_{4}\right)^{2} \cap\left(x_{2}, x_{3}, x_{4}\right)^{2}$. Then pd $S / I=3$. As all monomial prime ideals of height 3 , are among the associated radical ideals, depth $S(\mathfrak{p}) / I(\mathfrak{p})=0$, which implies $\operatorname{pd} S(\mathfrak{p}) / I(\mathfrak{p})=3$, for all $\mathfrak{p}$ with height $\mathfrak{p}=3$. Therefore, $I$ has stable projective dimension. Note that, $\sqrt{I}^{\vee}=$ $\left(\left(x_{1}, x_{4}\right) \cap\left(x_{2}, x_{3}\right)\right)^{\vee}=\left(x_{1} x_{4}, x_{2} x_{3}\right)$, does not have linear resolution. Therefore, $S / \sqrt{I}$ is not 
sequentially Cohen-Macaulay, by [4, Theorem 3.1]. Now, [10, Theorem 2.6], implies that $S / I$ is not sequentially Cohen-Macaulay.

The above examples, show that the class of monomial ideals $I$, where $S / I$ is sequentially Cohen-Macaulay and the class of ideals with stable projective dimension, are disjoint and non of them contains the other.

Proposition 1.17. Let $I \subset S$ be a monomial ideal with $\operatorname{Ass}(S / I)=\operatorname{Min}(I)$. Then $I$ has stable projective dimension if and only if $S / I$ is Cohen-macaulay.

Proof. If $S / I$ is Cohen-Macaulay, then obviously $I$ has stable projective dimension. Now if $S / I$ is not Cohen-Macaulay, we show that $I$ does not have stable projective dimension. Since $S / I$ is not Cohen-Macaulay, it follows that $\operatorname{pd} S / I>$ height $I$. Let $\mathfrak{p}^{\prime} \in \operatorname{Assh} S / I$ and $\mathfrak{p}=\mathfrak{p}^{\prime}+\mathfrak{q}$ be a monomial prime ideal with height $\mathfrak{p}=\operatorname{pd} S / I$. Since $\operatorname{Ass}(S / I)=\operatorname{Min}(I)$ and $\mathfrak{p}^{\prime} \subsetneq \mathfrak{p}$, it follows that $\mathfrak{p} \notin$ Ass $S / I$, so $\operatorname{depth} S(\mathfrak{p}) / I(\mathfrak{p}) \neq 0$. Therefore

$$
\operatorname{pd} S(\mathfrak{p}) / I(\mathfrak{p})=\operatorname{height} \mathfrak{p}-\operatorname{depth} S(\mathfrak{p}) / I(\mathfrak{p})=\operatorname{pd} S / I-\operatorname{depth} S(\mathfrak{p}) / I(\mathfrak{p})<\operatorname{pd} S / I .
$$

So $I$ does not have stable projective dimension.

Remark 1.18. The above result implies that for squarefree or unmixed monomial ideals (ideals $I$ with $\operatorname{Ass}(S / I)=\operatorname{Assh}(S / I)$ ), stable projective dimension property of $I$, is equivalent with Cohen-Macaulay property of $S / I$. Example 2.6, provides an equidimensional monomial ideal $I$ with stable projective dimension, such that $S / I$ is not Cohen-Macaulay.

The following result is a consequence of Proposition 1.17 and Remark 1.14.

Corollary 1.19. Let $I \subset S$ be a monomial ideal, such that $\operatorname{Ass}(S / I)=\operatorname{Assh}(S / I)$. Then the following statement are equivalent.

(a) I has stable projective dimension.

(b) $S / I$ is Cohen-Macaulay.

(c) $S / I$ is sequentially Cohen-Macaulay.

In the above corollary, (b) always implies (a) and (c), without any assumption on Ass $(S / I)$. The following example, shows that the condition $\operatorname{Ass}(S / I)=\operatorname{Assh}(S / I)$ is necessary for the inverse.

Example 1.20. Let $S=K\left[x_{1}, x_{2}, x_{3}\right]$ and $I=\left(x_{1}\right) \cap\left(x_{2}, x_{3}\right) \cap\left(x_{1}^{2}, x_{2}\right) \cap\left(x_{1}^{2}, x_{3}\right)$. Then $\operatorname{pd} S / I=2$. As all monomial prime ideals $\mathfrak{p}$ with height $(\mathfrak{p})=2$, belong to the associated prime ideals of $I, \operatorname{pd} S(\mathfrak{p}) / I(\mathfrak{p})=2$. In particular, $I$ has stable projective dimension. Since $\operatorname{dim} S=3$, the ideal $I$ is pretty clean by [13, Theorem 1.10]. Therefore, $S / I$ is sequentially Cohen-Macaulay by [7, Corollary 4.3].

\section{Polymatroidal ideals with stable projective Dimension}

In this section we characterize some special classes of polymatroidal ideals with stable projective dimension. Let $I \subset S=K\left[x_{1}, \ldots, x_{n}\right]$ be a monomial ideal generated in a single degree and $G(I)$ be the unique minimal set of monomial generators of $I$. Then $I$ is said to be polymatroidal, if for any two elements $u, v \in G(I)$ such that $\operatorname{deg}_{x_{i}}(u)>\operatorname{deg}_{x_{i}}(v)$ there exists an index $j$ with $\operatorname{deg}_{x_{j}}(u)<\operatorname{deg}_{x_{j}}(v)$ such that $x_{j}\left(u / x_{i}\right) \in I$. In the sequel a monomial prime ideal $\mathfrak{p}$ will be denoted by $\mathfrak{p}_{\left\{i_{1}, \ldots, i_{t}\right\}}$, where $\left\{i_{1}, \ldots, i_{t}\right\}=[n] \backslash\left\{x_{i} ; x_{i} \in \mathfrak{p}\right\}$.

Proposition 2.1. Let $I \subset S=K\left[x_{1}, \ldots, x_{n}\right]$ be a polymatroidal generated in degree 2 with at least one pure power $x_{i}^{2} \in I$, for some $1 \leq i \leq n$. Then I has stable projective dimension. 
Proof. Without loss of generality, we may assume that $I$ is fully supported. By [2, Proposition 2.1 (e)], after relabeling of the variables there exists integer $1 \leq k \leq n$ such that

$$
I=\left(x_{1}, \ldots, x_{k}\right)\left(x_{1}, \ldots, x_{n}\right)+J
$$

where $J$ is a squarefree monomial ideal in the variables $x_{k+1}, \ldots, x_{n}$. Set $\mathfrak{q}:=\left(x_{1}^{2}, x_{2}, \ldots, x_{n}\right)$. Obviously $\mathfrak{q}$ is one of the irreducible component of minimal primary decomposition of $I$. Hence $\mathfrak{m}=\sqrt{\mathfrak{q}} \in \operatorname{Ass}(S / I)$, and depth $S / I=0$. As $\mathfrak{m}$ is the only monomial ideal with height equal to $n=\operatorname{pd}(S / I), I$ has stable projective dimension.

The following example shows that the condition of having at least one pure power is essential.

Example 2.2. Let $I=\left(x_{1} x_{2}, x_{1} x_{3}\right) \subset K\left[x_{1}, x_{2}, x_{3}\right] . I$ is matroidal ideal generated in degree 2. As $S / I$ is not Cohen-Macaulay, $I$ does not have stable stable projective dimension by Remark 1.18.

An interesting special case of polymatroidal ideals is that of ideals of Veronese type. Given positive integers $d, a_{1}, \ldots, a_{n}$ such that $1 \leq a_{i} \leq d$. We let $I_{\left(d ; a_{1}, \ldots, a_{n}\right)} \subset S=K\left[x_{1}, \ldots, x_{n}\right]$ be the monomial ideal generated by the monomials $u \in S$ of degree $d$ satisfying $\operatorname{deg}_{x_{i}}(u) \leq a_{i}$ for all $i=1, \ldots, n$. Monomial ideals of this type are called ideals of Veronese type.

For the proof of the next theorem, we need the following results.

Lemma 2.3. [5, Theorem 3.3] Let $I=I_{\left(d ; a_{1}, \ldots, a_{n}\right)}$ with $1 \leq a_{i} \leq d$ for all $i$. Then

$$
\operatorname{pd} S / I=\min \left\{n, \sum_{i=1}^{n} a_{i}-d+1\right\} \text {. }
$$

Lemma 2.4. Let $J$ be a Veronese type ideal of $S$ and $\mathfrak{p}$ be a monomial prime ideal of $S$ with height $\mathfrak{p} \geq \operatorname{pd} S / J$. Then $\mathfrak{p} \in V^{*}(J)$.

Proof. Let $J=I_{\left(d ; a_{1}, \ldots, a_{n}\right)}$ with $1 \leq a_{i} \leq d$ for all $i$, and $\mathfrak{p}$ be a monomial prime ideal of $S$ with height $\mathfrak{p} \geq \operatorname{pd} S / J$. If $\operatorname{pd} S / J=n$, then $\mathfrak{m} \in \operatorname{Ass}(S / J)$ and height $\mathfrak{p} \geq n$. Hence $\mathfrak{p}=\mathfrak{m} \in V^{*}(J)$. Now let $\operatorname{pd}(S / J)=\sum_{i=1}^{n} a_{i}-d+1$. If $\sum_{i=1}^{n} a_{i}=d$, then $J=\left(x_{1}^{a_{1}} \cdots x_{n}^{a_{n}}\right)$. Since $a_{i} \geq 1$ for all $i \in[n], \mathfrak{p} \in V^{*}(J)$. Now assume that $\sum_{i=1}^{n} a_{i}>d$. We want to show that $\mathfrak{p} \in \operatorname{Ass}\left(S / J^{k}\right)$, where $k=$ height $\mathfrak{p}-1$, then obviously $\mathfrak{p} \in V^{*}(J)$. Since height $\mathfrak{p} \geq$ $\sum_{i=1}^{n} a_{i}-d+1$ and $\sum_{i=1}^{n} a_{i}>d$, we have that $k \geq 1$. By [8, Lemma 5.1], $J^{k}=I_{\left(k d ; k a_{1}, \ldots, k a_{n}\right)}$. Since $\sum_{i=1}^{n} a_{i}>d$, it follows that $k\left(\sum_{i=1}^{n} a_{i}-d\right) \geq k=$ height $\mathfrak{p}-1$. Hence $k\left(\sum_{i=1}^{n} a_{i}\right) \geq$ $k d-1+$ height $\mathfrak{p}$. On the other hand, Since $a_{i} \geq 1$, for all $i$, then

$$
\text { height } \mathfrak{p} \geq \sum_{i=1}^{n} a_{i}-d+1=\sum_{x_{i} \in \mathfrak{p}} a_{i}+\sum_{x_{i} \notin \mathfrak{p}} a_{i}-d+1 \geq \text { height } \mathfrak{p}+\sum_{x_{i} \notin \mathfrak{p}} a_{i}-d+1 .
$$

Hence $\sum_{x_{i} \notin \mathfrak{p}} a_{i}-d \leq-1$, so $k\left(\sum_{x_{i} \notin \mathfrak{p}} a_{i}-d\right) \leq-1$. Therefore by [8, Proposition 5.2], $\mathfrak{p} \in \operatorname{Ass}\left(S / J^{k}\right)$.

Theorem 2.5. Let $I=u J$ be a polymatroidal ideal of $S=K\left[x_{1}, \ldots, x_{n}\right]$, where $u$ is a monomial ideal and $J$ is a Veronese type ideal of $S$. Then I has stable projective dimension.

Proof. First, we show that if $I$ is a Veronese type ideal, then $I$ has stable projective dimension. Let $I=I_{\left(d ; a_{1}, \ldots, a_{n}\right)}$ with $1 \leq a_{i} \leq d$ for all $i$. If $\operatorname{pd} S / I=n$, then there is nothing to prove. Now let $\operatorname{pd} S / I=\sum_{i=1}^{n} a_{i}-d+1$. Let $\mathfrak{p} \in V^{*}(I)$ with height $\mathfrak{p} \geq \operatorname{pd} S / I$. We want to show that $\operatorname{pd} S / I=\operatorname{pd} S(\mathfrak{p}) / I(\mathfrak{p})$. We may assume that $\mathfrak{p}=\mathfrak{p}_{\{1, \ldots, k\}}$. Therefore, 
$n-k=$ height $\mathfrak{p} \geq \operatorname{pd} S / I$. We claim that $a_{j} \leq d-\sum_{i=1}^{k} a_{i}$ for all $j \notin[k]$. We have $\sum_{i=1}^{n} a_{i} \leq n-k+d-1$, because $\operatorname{pd} S / I \leq n-k$. Hence, since $a_{i} \geq 1$ for all $i$, it follows that

$$
a_{j}+n-k-1 \leq a_{j}+\sum_{\substack{k+1 \leq i \leq n \\ i \neq j}} a_{i}=\sum_{i=k+1}^{n} a_{i} \leq n-k+d-1-\sum_{i=1}^{k} a_{i}
$$

for all $j \notin[k]$. This implies that $a_{j} \leq d-\sum_{i=1}^{k} a_{i}$ for all $j \notin[k]$. Therefore, since $I(\mathfrak{p})=$ $I_{\left(d-\sum_{i=1}^{k} a_{i} ; a_{k+1}, \ldots, a_{n}\right)}$, it follows that $\operatorname{pd} S(\mathfrak{p}) / I(\mathfrak{p})=\min \left\{n-k, \sum_{i=1}^{n} a_{i}-d+1\right\}$, by Lemma 2.3. Hence $\operatorname{pd} S(\mathfrak{p}) / I(\mathfrak{p})=\sum_{i=1}^{n} a_{i}-d+1=\operatorname{pd} S / I$, because $\sum_{i=1}^{n} a_{i}-d+1 \leq n-k$.

Now we assume more generally that $I=u J$ is a polymatroidal, where $J$ is a Veronese type ideal of $S$. Let $\mathfrak{p} \in V^{*}(I)$ with height $\mathfrak{p} \geq \operatorname{pd} S / I$. Then since $\operatorname{pd} S / I=\operatorname{pd} S / J$, it follows that height $\mathfrak{p} \geq \operatorname{pd} S / J$ and also by Lemma 2.4, $\mathfrak{p} \in V^{*}(J)$. By the first part of the proof we know that $\operatorname{pd} S / J=\operatorname{pd} S(\mathfrak{p}) / J(\mathfrak{p})$. Hence $\operatorname{pd} S / I=\operatorname{pd} S(\mathfrak{p}) / I(\mathfrak{p})$, as desired.

Example 2.6. The Veronese type ideal $I=I_{(3 ; 2,2,1)} \subset S=K\left[x_{1}, x_{2}, x_{3}\right]$ has stable projective dimension. Although $I=\left(x_{1}^{2}, x_{2}\right) \cap\left(x_{1}, x_{2}^{2}\right) \cap\left(x_{1}, x_{3}\right) \cap\left(x_{2}, x_{3}\right) \cap\left(x_{1}^{2}, x_{2}^{2}, x_{3}\right)$ is equidimensional, it is not Cohen-Macaulay.

Let $I$ be a transversal polymatroidal ideal, say, $I=\mathfrak{p}_{1} \cdots \mathfrak{p}_{r}$ for monomial prime ideals $\mathfrak{p}_{1}, \ldots, \mathfrak{p}_{r}$. As in [8] we define the graph $G_{I}$ associated with $I$ as follows: the vertex set of $G_{I}$ is $[r]$ and $\{i, j\}$ is an edge of $G_{I}$ if and only if $G\left(\mathfrak{p}_{i}\right) \cap G\left(\mathfrak{p}_{j}\right) \neq \emptyset$.

The projective dimension of a transversal polymatroidal ideal is readable from its connected components:

Lemma 2.7. [8, Theorem 4.12] Let $I=\mathfrak{p}_{1} \cdots \mathfrak{p}_{r}$ be a transversal polymatroidal ideal, and let $H_{1}, \ldots, H_{s}$ be the connected components of $G_{I}$. Then

$$
\operatorname{pd} I=\sum_{j=1}^{s}\left(\left|\bigcup_{i \in H_{j}} G\left(\mathfrak{p}_{i}\right)\right|-1\right)=|\operatorname{supp}(I)|-s .
$$

Theorem 2.8. Let $I$ be a transversal polymatroidal ideal of $S=K\left[x_{1}, \ldots, x_{n}\right]$. Then $I$ has stable projective dimension if and only if one of the following conditions is satisfied:

(a) I is a product of principal ideals.

(b) I is the power of a monomial prime ideal.

(c) $G_{I}$ is connected and $I$ is fully supported.

Proof. Assume that $I$ has stable projective dimension. Let $I=\mathfrak{p}_{1} \cdots \mathfrak{p}_{r}$ and $H_{1}, \ldots, H_{s}$ be the connected components of $G_{I}$, and set $I_{j}=\prod_{i \in H_{j}} \mathfrak{p}_{i}$. We first show that if $\left|\mathfrak{p}_{i}\right| \geq 2$ for each $i \in[r]$, then $G_{I}$ is connected and $I$ is fully supported or $I$ is the power of a monomial prime ideal.

For each $j \in[s-1]$, we choose $i_{j} \in H_{j}$, and set $\mathfrak{p}=\mathfrak{p}_{\left\{i_{1}, \ldots, i_{s-1}\right\}}$. Since $\bigcup_{i \in H_{s}} G\left(\mathfrak{p}_{i}\right) \subseteq \mathfrak{p}$, it follows that $\mathfrak{p} \in V^{*}(I)$. Moreover, height $\mathfrak{p}=n-(s-1) \geq \operatorname{pd} S / I=|\operatorname{supp}(I)|-(s-1)$, see Lemma 2.7. Therefore, since $I$ has stable projective dimension, it follows that $\mathrm{pd} I=\operatorname{pd} I(\mathfrak{p})$.

Next we show that $\operatorname{pd} I_{j}(\mathfrak{p}) \leq\left|\operatorname{supp}\left(I_{j}\right)\right|-2$ for all $j \in[s-1]$. Indeed, if $I_{j}(\mathfrak{p})=S(\mathfrak{p})$ for some $j \in[s-1]$, then $\operatorname{pd} I_{j}(\mathfrak{p})=0$, and the assertion is trivial because $\left|\operatorname{supp}\left(I_{j}\right)\right| \geq 2$. On the other hand, if $I_{j}(\mathfrak{p}) \neq S(\mathfrak{p})$, then by using Lemma 2.7 , we obtain

$$
\operatorname{pd} I_{j}(\mathfrak{p})=\left|\operatorname{supp}\left(I_{j}(\mathfrak{p})\right)\right|-h \leq\left|\operatorname{supp}\left(I_{j}\right)\right|-1-h \leq\left|\operatorname{supp}\left(I_{j}\right)\right|-2,
$$


where $h \geq 1$ is the number of connected components of $G_{I_{j}(\mathfrak{p})}$. So in each case we have that $\operatorname{pd} I_{j}(\mathfrak{p}) \leq\left|\operatorname{supp}\left(I_{j}\right)\right|-2$ for all $j \in[s-1]$.

Since $I(\mathfrak{p})=\left(\prod_{j=1}^{s} I_{j}(\mathfrak{p})\right)$, it follows that $\operatorname{pd} I(\mathfrak{p})=\sum_{j=1}^{s-1} \operatorname{pd} I_{j}(\mathfrak{p})+\operatorname{pd} I_{s}$ because $G\left(I_{s}\right)=$ $G\left(I_{s}(\mathfrak{p})\right)$. Therefore, by using Lemma 2.7, we get

$$
\operatorname{pd} I=\operatorname{pd} I(\mathfrak{p}) \leq \sum_{j=1}^{s-1}\left(\left|\operatorname{supp}\left(I_{j}\right)\right|-2\right)+\left|\operatorname{supp}\left(I_{s}\right)\right|-1=\operatorname{pd} I-(s-1),
$$

which implies that $s=1$. Hence $G_{I}$ is connected.

Now, assuming that $I$ is not the power of a monomial prime ideal, we show that $I$ is fully supported. Suppose to the contrary that $|\operatorname{supp}(I)|<n$. Since $I$ is not the power of a monomial prime, there exist $1 \leq j, k \leq r$ and $x_{t} \in G\left(\mathfrak{p}_{j}\right) \backslash G\left(\mathfrak{p}_{k}\right)$. Let $\mathfrak{p}=\mathfrak{p}_{\left\{x_{t}\right\}}$. Then $\mathfrak{p} \in V^{*}(I)$ because $\mathfrak{p}_{k} \subset \mathfrak{p}$ and height $(\mathfrak{p})=n-1 \geq \operatorname{pd} S / I$.

Suppose $I(\mathfrak{p})=S(\mathfrak{p})$, then $0=\operatorname{pd} I(\mathfrak{p})=\operatorname{pd} I=|\operatorname{supp}(I)|-1 \geq 1$, a contradiction. On the other hand, if $I(\mathfrak{p}) \neq S(\mathfrak{p})$, then $\operatorname{pd} I(\mathfrak{p})=|\operatorname{supp}(I(\mathfrak{p}))|-d$, where $d \geq 1$ is the number of connected components of $I(\mathfrak{p})$. Hence $\operatorname{pd} I(\mathfrak{p}) \leq|\operatorname{supp}(I)|-1-d \leq|\operatorname{supp}(I)|-2=$ $\operatorname{pd} I-1<\operatorname{pd} I$, which is again a contraction. Thus we must have that $I$ is fully supported.

In order to conclude our proof that $I$ must satisfy one of the conditions (a),(b) or (c), it remains to be shown that if there exists $l \in[r]$, such that $\mathfrak{p}_{l}$ is principal, then $I$ satisfies one of the conditions (a) or (c). If $\mathfrak{m} \in \operatorname{Ass}(S / I)$, then $G_{I}$ is connected and $I$ is fully supported by [8, Theorem 4.3]. Otherwise, we show that $\mathfrak{p}_{i}$ is principal for all $i \in[r]$. If $\mathfrak{p}_{d}$ is not principal for some $d \in[r]$, then there exists $x_{t} \in \mathfrak{p}_{d} \backslash \mathfrak{p}_{l}$, and $\mathfrak{p}=\mathfrak{p}_{\left\{x_{t}\right\}} \in V^{*}(I)$, because $\mathfrak{p}_{l} \subset \mathfrak{p}$. Since $\mathfrak{m} \notin \operatorname{Ass}(S / I)$, hence $\operatorname{pd} S / I \leq n-1=\operatorname{height}(\mathfrak{p})$. We may assume that $\mathfrak{p}_{d}$ is one of the factors of the ideal $I_{1}$, as defined in the first part of the proof. Then $\operatorname{pd} I_{1}=\left|\operatorname{supp}\left(I_{1}\right)\right|-1 \geq 1$. If $I_{1}(\mathfrak{p})=S(\mathfrak{p})$, then $\operatorname{pd} I_{1}(\mathfrak{p})<\operatorname{pd} I_{1}$, and if $I_{1}(\mathfrak{p}) \neq S(\mathfrak{p})$, then by Lemma 2.7, pd $I_{1}=$ $\left|\operatorname{supp}\left(I_{1}\right)\right|-1>\left|\operatorname{supp}\left(I_{1}(\mathfrak{p})\right)\right|-1 \geq \operatorname{pd} I_{1}(\mathfrak{p})$. So in each case pd $I_{1}>\operatorname{pd} I_{1}(\mathfrak{p})$. Hence since $\operatorname{pd} I_{j} \geq \operatorname{pd} I_{j}(\mathfrak{p})$ for all $j \in[s]$, it follows that $\operatorname{pd} I=\sum_{i=1}^{s} \operatorname{pd} I_{i}>\sum_{i=1}^{s} \operatorname{pd} I_{i}(\mathfrak{p})=\operatorname{pd} I(\mathfrak{p})$, which contradicts our assumption that $I$ has stable projective dimension.

Conversely, it is obvious that $I$ has stable projective dimension in the cases (a) and (b). Now, assume condition (c) is satisfied. Then Lemma 2.7 implies that $\mathrm{pd} I=n-1$, and hence depth $S / I=0$, so $I$ has stable projective dimension.

The following examples, show that stability property of projective dimension is not inherited by radical and power.

Example 2.9. (a) Let $I=\left(x_{1}, x_{2}, x_{3}\right)\left(x_{1}, x_{4}\right) \subset S=K\left[x_{1}, \ldots, x_{4}\right]$. Since $I$ is connected and fully supported transversal polymatroidal ideal, it follows by Theorem 2.8 that $I$ has stable projective dimension, but $\sqrt{I}=\left(x_{1}, x_{2} x_{4}, x_{3} x_{4}\right)$ is not Cohen-Macaulay, so does not have stable projective dimension.

(b) Let $I=\left(x_{1} x_{2}, x_{2} x_{3}, x_{3} x_{4}\right) . S / I$ is Cohen-Macaulay and $\operatorname{Ass}\left(S / I^{2}\right)=\operatorname{Min}\left(I^{2}\right)$, but $S / I^{2}$ is not Cohen-Macaulay. So by Proposition 1.17, $S / I^{2}$ does not have stable projective dimension.

\section{ACKNOWLEDGMENTS}

The authors would like to thank Professor Jürgen Herzog for fruitful discussions and useful comments regarding this paper. 


\section{REFERENCES}

[1] J. Abbott, A.M. Bigatti and G. Lagorio, CoCoA-5: a system for doing Computations in Commutative Algebra. Available at http://cocoa.dima.unige.it 2, 3

[2] S. Bandari and J. Herzog, Monomial localizations and polymatroidal ideals, European J. Combin. 34 (2013), no. 4, 752-763. 7

[3] S. Bandari and R. Jafari, On certain equidimensional polymatroidal ideals, Manuscripta Math. 149 (2016), no. 1-2, 223-233. 4

[4] C. A. Francisco and A. Van Tuyl, Sequentially Cohen-Macaulay edge ideals, Proc. Amer. Math. Soc. 135 (2007), no. 8, 2327-2337. 5, 6

[5] J. Herzog and T. Hibi, The depth of powers of an ideal, J. Algebra 291 (2005), no. 2, 534-550. 7

[6] J. Herzog and T. Hibi, Monomial ideals, Graduate Texts in Mathematics, 260, Springer-Verlag London, Ltd., London, 2011. 2

[7] J. Herzog and D. Popescu, Finite filtrations of modules and shellable multicomplexes, Manuscripta Math. 121 (2006), no. 3, 385-410. 6

[8] J. Herzog, A. Rauf and M. Vladoiu, The stable set of associated prime ideals of a polymatroidal ideal, J. Algebraic Combin. 37 (2013), no. 2, 289-312. 7, 8, 9

[9] J. Herzog and E. Sbarra, Sequentially Cohen-Macaulay modules and local cohomology, in Algebra, arithmetic and geometry, Part I, II (Mumbai, 2000), 327-340, Tata Inst. Fund. Res. Stud. Math., 16, Tata Inst. Fund. Res., Bombay. 3

[10] J. Herzog, Y. Takayama and N. Terai, On the radical of a monomial ideal, Arch. Math. (Basel), 85(5) (2005), 397-408. 6

[11] H. Matsumura, Commutative ring theory, translated from the Japanese by M. Reid, Cambridge Studies in Advanced Mathematics, 8, Cambridge University Press, Cambridge, 1986. 2

[12] P. Schenzel, On the dimension filtration and Cohen-Macaulay filtered modules, in Commutative algebra and algebraic geometry (Ferrara), 245-264, Lecture Notes in Pure and Appl. Math., 206, Dekker, New York. 5

[13] A. Soleyman Jahan, Prime filtrations of monomial ideals and polarizations, J. Algebra 312 (2007), no. 2, 1011-1032. 6

Somayeh Bandari, Department of Mathematics, Buein Zahra Technical University, Buein Zahra, Qazvin, Iran and School of Mathematics, Institute for Research in Fundamental Sciences (IPM) P. O. Box: 19395-5746, Tehran, Iran.

E-mail address: somayeh.bandari@yahoo.com

Raheleh Jafari, Mosaheb Institute of Mathematics, Kharazmi University, Tehran, Iran

E-mail address: rjafari@ipm.ir 